\title{
Modernización y conflictos culturales: curación tradicional y asistencia médica entre nahuas en México - estudio de caso -
}

\section{György Szeljak}

Introducción. Uno de los problemas graves con que se enfrenta el Estado Mexicano hoy día, es cómo modernizar las comunidades indígenas marginadas con el objetivo de disminuir la tensión social provocada por la pobreza, la marginalidad, la escasez de la tierra, la superpoblación y los procesos migratorios incontrolables.

La intención modernizadora del Estado frecuentemente provoca discursos que reflejan las diferentes percepciones del mundo y los conocimientos culturales.

El objetivo de mi ensayo es analizar, con los métodos de la antropología cultural, cómo los habitantes de una comunidad nahua, en base a sus conceptos mitológicos y religiosos sobre las enfermedades y las curaciones, aceptaron e interpretaron las nuevas posibilidades que les ofreció la asistencia médica.

El lugar donde se desarolla el trabajo de campo es Pachiquitla, que se encuentra en México en la Huasteca hidalguense, en el municipio de Xochiatipan. La comunidad contaba en marzo de 2002 con 1.118 habitantes, agrupados en aproximadamente 300 familias. Los habitantes son campesinos nahuahablantes y autosubsistentes en alto grado. Cultivan sus tierras ejidales con herramientas tradicionales, siembran sobre todo maíz, chile, frijol, café y caña de azúcar. La comunidad en los últimos años ha experimentado un alto grado de migración hacía México D.F.

El antecedente de la inauguración de la clínica rural en Pachiquitla fue una epidemia de cólera en 1993. En unas semanas se enfermaron más de 100 personas y 7 fallecieron. A consecuencia de esto, en 1996, inauguraron una clínica rural. Un médico y dos enfermeras empezaron sus actividades, respondiendo a las demandas de la comunidad.

Antes de esa fecha los vecinos caminaban de tres a cuatro horas por las veredas empinadas entre las montañas, llevando en una silla fija a sus espaldas a los enfermos graves hasta la clínica desde la cabecera municipal ubicada a unos 6 kilometros. Por falta de confianza en la asistencia médica, la mayor parte de las familias ni siquiera buscaban la ayuda del médico municipal, sino que acudían a los especialistas locales, o ellos mismos intentaban curarse con yerbas medicinales.

Asistencia médica en Pachiquitla. En la región, las clínicas rurales forman el peldaño más bajo en la jerarquía de asistencia médica. Es el nivel preventivo. Aparte de localizar las enfermedades y buscar la medicina efectiva para la curación, los médicos y sus ayudantes están obligados a hacer una labor intensiva de convencimiento hacia a la población. ${ }^{1}$ En Pachiquitla ellos, junto con los profesores, son los mediadores más importantes entre la comunidad marginada y el Estado-Nación, para impulsar procesos de nacionalización, cambios culturales y propagar un nuevo estilo de vida. En este sentido la asistencia médica es considerada como un elemento importante de control social del Estado-Nación. Por supuesto, todo lo anterior no quiere decir que los médicos y las enfermeras realizaran un proceso consciente de aculturación agresivo. Según sus mejores intenciones y conocimientos de la tradición occidental de medicina, (basada en la ciencia, la racionalidad y la objetividad académica) intentaron curar a los enfermos, luchar contra el alcoholismo, propagar un consumo más abundante en vitaminas y proteínas, u ofrecer una nueva interpretación sobre la higiene y la limpieza del ambiente para los vecinos.

El médico y sus asistentes, en los primeros años de sus actividades en la comunidad, se enfrentaron a varios tipos de conflictos, mientras intentaban establecer su legitimización social. Trabajaban en un ambiente cultural que no era propio de ellos, donde los vecinos ya tenían sus conceptos tradicionales sobre las enfermedades, sus fondos mitológicos y religiosos sobre las

\footnotetext{
${ }^{1}$ Los aspectos espaciales y estructurales del funcionamiento del sector de la salud mexicano véase Margaret E. HARRISON, "Mexican Physician, Nurses and Social Workers", in: Brad R. Huber - Alan R. Sandstrom (eds.) Mesoamerican Healers, Austin, University of Texas Press, 2001. 270-306. y Margaret SHERRARD SHERRADEN, "Policy Impacts of Community Participation: Health Services in Rural Mexico", in Human Organization, Vol. 50. No. 3, 1991. 256-263.
} 
curaciones efectivas. Los habitantes, en base a esta lógica cultural, se acercaron a la medicina occidental para aprovecharla, pero esperaban que el médico y las enfermeras entendieran los conceptos locales.

Enfermedades e interpretaciones locales. La interpretación de las enfermedades y los métodos de curación son construcciones culturales, las cuales varían en diferentes ambientes sociales y culturales.

Como Kleinman plantea: "los sistemas de atención a la salud son sistemas simbólicos construidos por significados, valores y normas de conducta, que articulan al padecimiento como un idioma cultural, uniendo las creencias sobre la causalidad de la enfermedad, la experiencia de los sintomas, los patrones especificos de la conducta frente al padecer, las decisiones respecto de los tratamientos alternativos o las prácticas terapéuticas vigentes y la evalución de los resultados terapéuticos y estableciendo relaciones sistemáticas entre estos componentes". ${ }^{2}$

Los nahuas en Pachiquitla no vinculan directamente al cuerpo las causas principales de las enfermedades y del fallecimiento. La mal función del cuerpo es solamente la consecuencia de la debilidad o la disfunción de los componentes anímicos de los individuos. Aunque a consecuencia de la conversión cristiana este concepto se mezcló con el concepto católico del alma, los nahuas incluso hasta hoy interpretan las enfermedades según el modelo prehispánico. ${ }^{3}$ En Pachiquitla se distinguen dos partes del alma: el yolotl y el tonalli. ${ }^{4}$ El yolotl - literalmente es el corazón - es la fuerza vital impersonal y está dentro del cuerpo siempre y cuando el corazón funcione. La otra parte del alma es el tonalli, que los nahuas consideran como un alma segmentada personal, asociada al calor del sol, del cuerpo, al destino y suerte y a la personalidad individual. Es una entidad invisible que según los pobladores de esta zona puede encontrarse en cualquier parte del cuerpo, sobre todo en la cabeza, y en los puntos donde perciben el trabajo rítmico de las venas.

Aunque sea una enfermedad natural o causada por seres sobrenaturales, si no se encuentra el tratamiento adecuado, el tonalli se debilita o sale del cuerpo y la enfermedad llega hasta el corazón y el enfermo puede llegar a la muerte.

Según las interpretaciones locales las enfermedades mágicas más importantes son el susto (nemahtili) o la pérdida del tonalli, la intrusión de malos aires o vientos (ajakatl) al cuerpo, y las enfermedades causadas por la actividad maligna de los brujos (tetlachiuijketl).

Cualquier experiencia emocional extraña puede causar el susto. En este caso el tonalli sale del cuerpo, los guardianes de los sitios naturales (la tierra madre, los espíritus de los arroyos, ríos, cerros) lo atrapan y no dejan que regrese al cuerpo.

Los malos aires son seres invisibles, que viven en el ambiente natural alrededor de la comunidad. Por propia voluntad, por intención de los guardianes de los sitios o por las de los brujos son capaces de entrar al cuerpo, debilitar al tonalli, y matar al enfermo.

Según los nahuas, los brujos son especialistas malignos con dones mágicos que recibieron del diablo. Siempre trabajan en secreto. Por su propia voluntad o por encargo de otra persona son capaces de hacer enfermar a cualquier otra con magia simpatética, mandando malos aires al cuerpo de alguien o asustándole. En el modelo general de las interpretaciones de la brujería la causa es siempre la envidia por la riqueza, la buena cosecha, la suerte o cualquier cosa extra que alguien tenga en la comunidad. Nadie se identifica como brujo, pero los curanderos continuamente tienen que defenderse contra las acusaciones.

\footnotetext{
${ }^{2}$ Tomé la cita del estudio de Rosa Maria OSORIO CARRANZA,.Entender y atender la enfermedad. Los saberes maternos frente a los padecimientos infantiles. Mexico, INI-CIESAS-INAH, 2001. 23.

${ }^{3}$ Sobre los conceptos prehispánicos de las entidades anímicas entre nahuas del Altiplano Central de México véase: Alfredo LÓPEZ AUSTIN, Cuerpo humano e ideología, México, UNAM 1989, 221-262.

Sobre los intentos de los frailes para acordarlos con las ideas católicas después de la conquista véase Luise M. BURKHART, The slippery Earth. Nahua-Christian Moral Dialogue in Sixteenth-Century Mexico, Tucson, The University of Arizona Press 1989. La sobrevivencia de estos conceptos entre los diferentes grupos nahuas contemporaneos véase: Italo SIGNORINI y Alessandro LUPO, Los tres ejes de la vida. Almas, cuerpo, enfermedad entre los nahuas de la Sierra de Puebla, Xalapa, Universidad Veracruzana 1989, 46 - 81. y Alan SANDSTROM, Corn is Our Blood. Culture and Ethnic Identity in a Contemporary Aztec Indian Village. Norman and London, University of Oklahoma Press 1991, 257-260.

${ }^{4}$ En el ensayo indicaré con letras cursivas las palabras nahuatl.
} 
Curación simbólica y conflictos culturales. En Pachiquitla -aparte de la autocuración por medio de yerbas medicinales y rezos dentro de la unidad familiar- los terapeutas tradicionales responsables de la salud de los vecinos eran las parteras (teteketl) y los curanderos tradicionales (tlamatiketl). Ellos también curan con yerbas medicinales, soban a los pacientes, y varios de ellos actúan como hueseros. ${ }^{5}$ Pero su actividad principal es la curación simbólica a través de los ritos curativos.

Como James Dow apunta, la curación simbólica puede alcanzar su objetivo si en la cultura local existe un modelo general del mundo mítico en que tanto el terapeuta como el paciente creen. ${ }^{6}$ Durante la práctica de los ritos de curación los curanderos particularizan e invocan una parte de este mundo mítico e incluyen en la realidad la enfermedad del paciente. ${ }^{7}$

El objetivo de la curación por los especialistas tradicionales es encontrar el equilibrio con los malos aires, con los santos católicos y sobre todo con los guardianes de los sitios naturales. Como Gréco menciona, los guardianes o dueños de los sitios naturales "controlan el uso de la naturaleza por parte de los hombres. (...) castigan la falta de respeto a los lugares y el incumplimiento de la regla del intercambio. (...) La curación implica entonces reconciliarse con estos seres". 8

Durante los ritos de curación, los especialistas forman un espacio y un tiempo sagrado con la ayuda de los símbolos rituales (rezos, velas, copal, flores, comida, bebida, tabaco, muñecos de papel), donde la interacción con ellos se puede realizar en un ambiente de seguridad. La interacción nunca es la confrontación agresiva -aunque ellos sean capaces de matar al enfermo- sino que siempre está basada en la práctica ritual del respeto y la reciprocidad.

Aunque los seres sobrenaturales son invisibles, se les considera como antropomorfos, les gusta comer, beber, fumar cigarros y divertirse junto con los vivos. Como trabajan, se cansan, y el mejor método de gozar de su favor es ofreciéndoles ofrendas.

Los ritos de curación se realizan en un ambiente cultural y natural conocido por el enfermo (en la casa del terapeuta o del enfermo, en las cimas de los cerros sagrados alrededor de la comunidad o en ciertos lugares, donde el enfermo perdió su alma). En la preparación de los objetos rituales participan los familiares, quienes ayudan activamente durante los ritos. Aunque los conocimientos sobre la mitología y sobre los significados de los símbolos varían, una tarea importante de los especialistas es la interpretación adecuada a través de la consulta, de los rezos y de los usos de los símbolos durante el proceso ritual.

Al inaugurar la clínica rural, el médico y sus ayudantes hicieron enormes esfuerzos para convencer a la gente a aceptar sus servicios. Se enfrentaron con varios problemas. El ambiente de la clínica estaba fuera del ambiente conocido por los habitantes. Sin la presencia de los familiares tenían que presentarse ante el médico. Sobre todo a las mujeres les causó problemas emocionales, el tener que desvertirse en el consultorio frente a un hombre desconocido o en problemas graves tener que pasar la noche sola en la sala de hospitalización. Los instrumentos que usa el médico (por ejemplo el estetoscopio, el termómetro) tampoco les daban una seguridad emocional y no eran capaces de interpretarlos e incluirlos entre los símbolos de curación conocidos culturalmente por ellos. Además, por las diferencias culturales y por la falta de términos adecuados, no podían expresarse de tal manera que el médico entendiera. Y al revés, el médico manejó un lenguaje técnico. No hacía referencia a la cultura, a la cosmología, ni al mundo mítico, sus consejos y explicaciones solamente se referían a los problemas del cuerpo, a la alimentación o a la higiene insuficiente.

\footnotetext{
${ }^{5}$ El uso de las yerbas medicinales durante el proceso curativo en la Huasteca hidalguense véase: Danielle GRÉCO, "Notas para el estudio de la medicina tradicional en una comunidad náhuatl de la Huasteca hidalguense", in: Jesús Ruvalcaba - Graciela Alcalá (coords.) Huasteca II. Prácticas agrícolas y medicina tradicional. Arte y sociedad, México, CIESAS 1993, 51-73.

${ }^{6}$ James DOW, "Universal Aspects of Symbolic Healing” American Antropologist 88 (1) 1986, 60.

${ }^{7}$ En este ensayo breve no tenemos lugar de analizar detalladamente la cosmovisión de los nahuas de la Huasteca y los diferentes ritos de curaciones. Sobre este tema véase por ejemplo GRECO op. cit., SANDSTROM op.cit., Rosendo HERNÁNDEZ CUELLAR, La religión nahua en Texoloc, municipio de Xochiatipan, Hgo. Etnolinguística 51. México D.F., SEP-INI. 1981.; Arturo GÓMEZ MARTÍNEZ, Tlaneltokilli. La espiritualidad de los nahuas chicontepecanos. Mexico, Programa de Desarollo Cultural de la Huasteca. 2002.

${ }^{8}$ GRÉCO, op. cit., 51.
} 
Lo anterior no significa que los habitantes no participaran en las consultas. Según el registro del médico, en 1997 atendió 2.063 consultas en la comunidad, que contaba en ese tiempo con 1.196 habitantes permanentes. ${ }^{9}$ Los nahuas de Pachiquitla no son fatalistas o fundamentalistas religiosos. En una sociedad mayoritariamente de autoconsumo y sin recursos suficientes, la falta de mano de obra a causa de las enfermedades provoca una crisis grave en la familia. Aunque ellos clasificaban las enfermedades como naturales o causadas por seres sobrenaturales o por brujería, primero usaban estrategias conscientes y esperaban las diferentes interpretaciones y métodos sobre la curación adecuada.

Ellos acudían al médico que les ofrecía gratis su servicio, y si él no era capaz de curar la enfermedad (es decir, la raíz de la enfermedad no era natural), buscaban un curandero reconocido, o viceversa, dependiendo de las familias.

Además, durante las asistencias médicas también intentaron usar una estrategia consciente basada en sus lógicas culturales. Como mencioné anteriormente, en Pachiqitla la causa principal de la mayoría de las enfermedades es la debilidad del tonall o alma. El tonalli aumenta la fuerza de la sangre, que los nahuas llaman chicaualistli, y lo perciben como el calor óptimo del cuerpo. Quien tiene sangre más densa, tiene un tonalli más fuerte para caminar, trabajar y mantener a su familia. Durante las consultas médicas muchos vecinos protestaron y no querían que el médico les sacara sangre para un análisis y poder diagnosticar ciertos tipos de enfermedades. Se opusieron especialmente las madres embarazadas y las que tenían niños pequeños. Sus almas se consideraron más débiles y así las enfermedades causadas por malos aires o brujería podían atacarles más fácilmente.

El método tradicional para asegurar la fuerza vital de la sangre es comer suficiente comida, sobre todo maíz, la planta sagrada que les regalaron los dioses. Vivir una vida socialmente aceptada, y dejar ofrendas en los lugares sagrados disminuye también el peligro de la brujería y el ataque de los seres sobrenaturales. Según los vecinos de Pachiquitla ciertos métodos de la curación occidental también son capaces de aumentar este tipo de fuerza.

Por ejemplo la mayoría de los pacientes preferían inyecciones, y en situaciones graves incluso infusiones en vez de pastillas, aunque el médico intentaba explicarles que las pastillas y las inyecciones tienen la misma efectividad. El motivo para rechazar las pastillas era que su consumo exigía cierto tipo de regularidad temporal. Pero una causa más importante era que muchos habitantes no consideraban que las diferentes pastillas e inyecciones fueran los tratamientos adecuados para los diferentes tipos de enfermedades. Según las interpretaciones locales, las pastillas e inyecciones contienen ciertas cantidades de energía o fuerza vital que ayuda al enfermo a tener suficiente cantidad y densidad de sangre y fuerza. Las inyecciones y las infusiones entran directamente y en cantidades concentradas al torrente sanguíneo, donde se mezclan con la sangre, y refuerzan el tonalli de la persona.

Después de acudir al médico y recibir las inyecciones, los pobladores según el concepto del síndrome caliente-frío, se abstenían de comer lo que ellos consideran del tipo frío. ${ }^{10}$ Tampoco participaron en los velorios, donde el alma fría y enojada del muerto les hubiera podido causar algún daño.

Los partos. Los miembros de la asistencia médica hicieron esfuerzos para que las mujeres estuvieran bajo control médico durante sus embarazos y dieran a luz a sus bebés en un ambiente estéril y seguro bajo la vigilancia del médico. Los institutos estatales de salud también les exigieron alcanzar una mejor estadística de un año para otro. A pesar de todas sus intenciones, desde 1996

\footnotetext{
${ }^{9}$ Próspero HERNÁNDEZ HERNÁNDEZ - Magdalena DE LA CRUZ MARTÍNEZ - Eva TORRES ONTIVEROS, Diagnostico de salud de la comunidad de Pachiquitla, Mpio. de Xochiatipan, Hgo, tesis para obtener el título de profesional técnico en atención primaria a la salud. Pachuca de Soto, 1998, 27.

${ }^{10}$ El concepto del síndrome caliente-frío en las interpretaciones sobre las enfermedades véase Diana RYESKY, Conceptos tradicionales de la medicina en un pueblo mexicano, Mexico, Sepsetentas 1976, 32-65. y George FOSTER, "The validating role of humoral theory in traditional Spanish-American therapeutics", in: American Ethnologist, Vol. 15, No. 1, 1988, 120-135.
} 
hasta 2002, más de ciento veinte partos se realizaron en la comunidad con la ayuda de la medicina tradicional, solamente en dos casos se le pidió ayuda al médico en situaciones críticas. ${ }^{11}$

Entre los nahuas los partos son uno de los ritos de paso más importante durante la vida. Estos siempre se realizan en el espacio sagrado de la casa familiar, cerca del altar, donde solamente los familiares muy cercanos tienen derecho a participar. La partera es la que durante el proceso del embarazo cuida a la madre y al feto, la soba, le hace limpias durante visitas periódicas. Ella está presente continuamente durante el parto, prende velas y copal en el altar para sacralizar el espacio. Mientras que da instrucciones a la mujer reza a la Virgen de Guadalupe o a Tonantzin y usa yerbas curativas para aliviar el dolor de la mujer e intenta que el parto sea sencillo. ${ }^{12}$ Durante el parto la mujer pierde mucha sangre, su tonalli queda muy débil. Asimismo el tonalli del niño recién nacido también es débil.

La partera pone yerbas medicinales en las cuatro esquinas de la casa, en las puertas y en las ventanas, para que los malos aires no puedan entrar al hogar. Además la partera es quien lava la ropa ensangrentada de la mujer, y baña al niño por la noche, cuidándole de los malos aires y de la brujería.

Según los vecinos, dar a luz en la clínica es muy peligroso. No hay altar, el médico y las enfermeras no conocen los rezos tradicionales ni el uso de los otros símbolos adecuados, por eso no son capaces de formar un espacio sagrado y seguro, ni de tener contactos con los seres sobrenaturales.

La actividad de las enfermeras se redujo a obligar a las 5 parteras tradicionales a capacitarse, yendo una vez al mes al centro de salud, y dándoles utensilios estériles para que los utilizaran durante los partos. Pero después de unos días del parto, las mujeres aceptaban las visitas de las enfermeras y vacunaban a los bebes, para que el tonalli del niño se reforzara.

Argumentos culturales y planificación familiar. Además del parto, el discurso más intenso entre los vecinos y los miembros del centro de salud era la propagación de la planificación familiar. Les dieron gratis pastillas e inyecciones anticonceptivas a las mujeres, e intentaron convencer a las madres que ya tenían varios hijos, que pidieran la operación de esterilización en el hospital de la ciudad Atlapexco. Según los argumentos de las instituciones estatales, la planificación familiar es una forma efectiva de combatir la pobreza en las comunidades indígenas, obstaculizar los conflictos locales por la escasez de la tierra y evitar la migración. Según ellos la tendencia a tener muchos hijos es una de las causas por la cual los indígenas no son capaces de llevar a la práctica la estrategia económica impulsada por el Estado y sus institutos, es decir, la acumulación e inversión del poco dinero que tienen, el mejoramiento de sus niveles de vida, la escolarización de sus hijos y el desarollo social a través de las generaciones. ${ }^{13}$

Antes de la inauguración de la clínica no contamos con datos precisos sobre la natalidad y la mortalidad de la población. En 1997 en la comunidad nacieron 26 niños y fallecieron 5 personas. El año siguiente nacieron 39 niños y murieron 4 personas, lo que muestra que la proporción entre la mortalidad y el nacimiento es desequilibrada.

El argumento de los discursos de la modernización se enfrentaron con los argumentos culturales de los nahuas. En la sociedad pachiquitlense el número de hijos que una familia tenga es una forma efectiva de alcanzar respeto y estatus dentro de la comunidad. Además, los ritos de paso de los niños (bautismo, primera comunión, fiestas de graduación, bodas) ofrecen una importante oportunidad de crear redes de solidaridad entre las familias, por los compromisos del compadrazgo, que son indispensables durante los trabajos de temporal y en las situaciones de crisis de la vida.

\footnotetext{
${ }^{11}$ Comunicación personal con el médico de la comunidad.

${ }^{12}$ Tonantzin o "nuestra madrecita" era la diosa de la fertilidad entre los aztecas. A consecuencia de la conversión católica los rasgos de la diosa se sincretizaron con los rasgos de la Virgen de Guadalupe. Hoy día los nahuas en Pachiquitla los consideran como la misma diosa quién es responsable por la fertilidad de las mujeres.

${ }^{13}$ En 1900 la población estimada de México era 13,6 millones, mientras que en 1997 llegó hasta el 95,7 millones. Asimismo la expectación de vida subió de 29,5 años (1900) a 72,6 años (1997). Entre las causas del crecimiento rápido de la población nos encontramos el mejoramiento de la vida económica y social y el desarollo del sector de la salud en el país. Véase: HARRISON, op. cit., 271-273.
} 
Además, dentro de la costumbre, la fertilidad y la reproducción son conceptos complejos que incluyen a la naturaleza, a la milpa que cultivan las familias y a la fertilidad de la mujer. Todos nacemos de la tierra, la tierra nos alimenta y cuando morimos regresamos a la tierra madre - como me comentó un curandero. Después del parto entierran el cordón umbilical del recién nacido en la milpa, tarea que realiza el padre. Lo entrega a la tierra madre que los alimenta a todos ellos, luego siembra un brote de plátano sobre ella.

Dentro de la cultura tradicional es una obligación moral dar a luz a los bebés. Si una mujer fértil no quiere quedarse embarazada, su mentalidad choca directamente con las costumbres centrales: con el respeto y con la reciprocidad. En este caso la tierra tampoco queda fértil, y pueden aparecer enfermedades entre los miembros de la familia. Según el pensamiento nahua tradicional, la futura seguridad de la familia depende principalmente de la reproducción y de la armonía creada con la naturaleza y con los seres sobrenaturales y, menos, de la acumulación económica.

Parece que la oposición a la planificación familiar controlada se refuerza con los principios católicos referentes a los métodos anticonceptivos. Además, el valor de la maternidad se manifiesta también - como Wolf y Campbell mencionan - en la costumbre y en la religión católica mexicana, donde Tonantzin o la Virgen de Guadalupe desempeña un papel muy importante con respecto a la fertilidad femenina. ${ }^{14}$.

A pesar de los argumentos culturales, la planificación familiar, primero lentamente y luego un poco más aceleradamente, empezó a ganar terreno en la comunidad. Muchas los pidieron en secreto, sin el consentimiento de sus maridos u otros familiares. Además en este tiempo el problema provocó varios conflictos intensos dentro de la comunidad y la unidad familiar.

En agosto de 2002 el número de mujeres que planificaban su familia incrementó hasta el 25-30 por ciento. ${ }^{15}$ La causa del incremento - aparte de la labor de convencimiento permanente de las enfermeras - es por un lado el regreso de los migrantes de la comunidad con nuevas ideas sobre la familia, y sobre la acumulación económica. Y por otro la instalación de la electricidad y la aparición de las televisiones. Los mensajes de los anuncios comerciales y estatales, y sobre todo las telenovelas, lentamente empezaron a influir en la opinión de las familias. Las telenovelas podemos considerarlas como los mensajes indirectos del Estado que ofrecen un nuevo modelo de vida para las jóvenes y mujeres maduras. En estas telenovelas las mujeres son presentadas como más emancipadas, su primer objetivo no es casarse tan jóvenes, ni tener muchos niños. Además, a través de los programas de televisión, los receptores interpretan, en un contexto social más amplio, su propia pobreza, reforzando la migración y las nuevas estrategias económicas.

Conclusiones. Esta investigación se enfocó principalmente en los resultados de los primeros años de la presencia médica institucional en dicha comunidad marginada. Intenté mostrar algunos conflictos y métodos de adaptación entre las diferentes lógicas acerca de las enfermedades y las curaciones. Supuestamente con una mayor integración de la comunidad a la sociedad nacional contemporánea, estos tipos de discursos entre los pobladores y los médicos cambiarían paulatinamente.

El intento de las instituciones de salud es homogeneizar las diferencias culturales con la introducción de nuevos conceptos referentes a este tópico, y crear un ambiente cultural más propicio para tal problemática, en donde las diferentes lógicas culturales no obstaculicen el éxito de la curación basada en la experiencia científica occidental; que se enfoca principalmente en los problemas del cuerpo. Además, en el fondo de estos intentos de homogeneización, podemos develar el intento del Estado-Nación, para efectuar un control social eficaz sobre la población.

Pero ¿qué hacen los nahuas en Pachiquitla? Normalmente usan estrategias conscientes para mantener la salud de sus familias, acudiendo a los diferentes especialistas y mezclando los métodos de curación tradicionales con los occidentales. En este contexto se podría hablar más de un proceso de hibridación consciente que de homogeneización. En particular considero que lo más importante

\footnotetext{
${ }^{14}$ Eric R. WOLF, “The Virgin of Guadalupe: A Mexican National Symbol”, in: Lessa, W - Vogt, E. (ed.) Reader in Comparative Religion, New York, Harper and Row 1958, 226-230; Ena CAMPBELL "The Virgin of Guadalupe and the Female Self Image: A Mexican Case History. Preston, J. (ed.) Mother Worship: Theme and Variations, Chapel Hill:University of North Carolina Press 1982, 5-25.

${ }^{15}$ Conversación personal con el médico de Pachiquitla.
} 
sería que el Estado mexicano apoyara con firmeza a este tipo de comunidades marginadas con mayores oportunidades para que pudieran obtener suficientes recursos económicos y así, desarrollar una cotidianidad rural más saludable, eligiendo libremente entre los métodos de curación o la mezcla de estos que ellos consideren los más adecuados.

Normalmente los argumentos culturales conservan mayor énfasis en los estudios antropológicos, mientras que los médicos enfatizan en los derechos individuales para tener una vida más prolongada y saludable en base a la medicina occidental. Sin embargo, no creo que sea una lucha entre buenos y malos al estilo hollywoodiense. Lo más importante sería que el sector salud profundice sus conocimientos del contexto cultural de las enfermedades y sus explicaciones sean más adecuadas al ambiente social y cultural. En este sentido, las investigaciones antropológicas en conjunto con los diversos agentes institucionales lograrían una solución más determinante para tal problemática. 